\title{
Trauma, Multiple Sclerosis, Delayed Sleep Phase Disorder, Subjective Experiences, and Duration of Illness in GAD
}

\author{
By Eric Hollander, MD
}

In this month's issue we launch the new series, Grand Rounds. The first installment is from a presentation at the Mount Sinai School of Medicine. In it, Noah DeGaetano, MD, describes a fascinating case of posttraumatic stress disorder (PTSD) with an unusual presentation and discusses the case from various formulations, including a psychodynamic perspective. The discussant, Rachel Yehuda, PhD, questions whether making a diagnosis of PTSD helps in the healing, explores the true source of the trauma, the impact of earlier attachment issues, and the use of cognitive techniques to accelerate treatment response in this patient. We hope that this series eventually spreads to other academic and international departments of psychiatry and neurology.

Pain, spasticity, tremor, spasms, poor sleep quality, and bladder and bowel dysfunction, among other symptoms, contribute significantly to the disability and impaired quality of life of many patients with multiple sclerosis (MS). Motor symptoms referable to the basal ganglia, especially paroxysmal dystonia, occur rarely and contribute to the experience of distress. A substantial percentage of patients with MS report subjective benefit from what is often illicit abuse of extracts of the Cannabis sativa plant; the main cannabinoids include delta-9-tetrahydrocannabinol $\left(\Delta^{9}-\mathrm{THC}\right)$ and cannabidiol. Clinical trials of cannabis plant extracts and synthetic $\Delta^{9}$-THC provide support for therapeutic benefit on at least some patient self-report measures. Stephen I. Deutsch, MD, PhD, and colleagues describe a case of a 52-year-old woman with MS, paroxysmal dystonia, complex vocal tics, and marijuana dependence. The patient was started on a trial of dronabinol, an encapsulated form of synthetic $\Delta^{9}-T H C$ that is usually prescribed as an adjunctive medication for patients undergoing cancer chemotherapy. She reported a dramatic reduction of craving and illicit use, and did not experience a "high". The patient also reported an improvement in the quality of her sleep with diminished awakenings during the night, decreased vocalizations, and the tension associated with their emission, decreased anxiety and a decreased frequency of paroxysmal dystonia. Clearly, the risks versus benefits of such an approach need to be studied in more systematic trials, but this case is informative.

Suman Mukhopadhyay, MRCPsych, and colleagues studied the prevalence of delayed sleep phase (DSP) in a cohort of inpatients with severe obsessive-compulsive disorder (OCD) to identify clinical and demographic correlates. Out of 187 patients, $33(17.6 \%)$ fulfilled criteria for DSP after exclusion of possible confounding factors. All the patients with DSP were unemployed. Phaseshifted patients were significantly younger than non-shifted patients and reported an earlier age of onset of their OCD. There was a non-significant trend toward more severe OCD in the phaseshifted group, but they were not more depressed than their non-shifted counterparts. Thus, a substantial number of patients with severe, enduring OCD also suffer with DSP, which seems to be specifically linked to $O C D$ as opposed to comorbid depression. Clarification of the etiology within

Dr. Hollander is the editor of this journal, Esther and Joseph Klingenstein Professor and Chairman of Psychiatry at the Mount Sinai School of Medicine, and director of the Seaver and New York Autism Center of Excellence in New York City. 
DSP and its interaction with core OCD symptoms on clinical function and disability may potentially identify new treatment targets.

A. Carlo Altamura, MD, and colleagues studied the impact of the duration of untreated illness (DUI)-the time elapsing between the onset of generalized anxiety disorder (GAD) and the first adequate pharmacologic treatment-on treatment response and clinical course in a sample of 100 subjects with GAD. For antidepressant treatment, shorter DUI was associated with greater improvement. In contrast, for benzodiazepines, DUI did not influence treatment response. Patients with a longer DUI showed an earlier age at onset, a longer duration of illness, and a higher rate of comorbid psychiatric disorders with onset later than GAD. This study suggests that a shorter DUl for antidepressants may determine a better response in patients with GAD, and that a longer DUI may be associated to a worse clinical course. This is of interest, since discussions regarding the Diagnostic and Statistical Manual of Mental Disorders, Fifth Edition will explore whether GAD and major depression lie on a continuum, especially since untreated GAD often leads to depression.

Helena da Silva Prado, MSc, and colleagues describe obsessive-compulsive symptoms and tics as core clinical features of $O C D$ and Tourette syndrome (TS) but note that a variety of subjective experiences have also been reported in these disorders. Patients with OCD and/or TS sometimes report that the subjective experiences may cause more distress than the compulsions or tics themselves. The presence of these subjective experiences may be helpful to identify OCD patients with early onset or with OCD associated with tics. Also, a better recognition of the presence of these subjective experiences may increase the patients' ability to suppress symptoms, and the presence of subjective experiences may be a predictive factor of treatment response. Studies have been limited by the fact that there is no consensus in the literature on how to best define them. However, this article suggests such a consensus definition.

This month's CNS Spectrums provokes new ways to think about common disorders and potentially new targets for treatment in such conditions. By intervening earlier in the course of GAD; identifying subjective experiences or delayed sleep phase in OCD or TS patients; or using case reports of efficacy to guide further clinical trials in MS, we may alter the way we see and treat these diverse patient populations. CNS 